\title{
Neurosarcoidosis: An Overlooked Diagnostic Dilemma
}

\section{Suneel Kumar ${ }^{1}$, Saeed Ahmed ${ }^{2}$, Savita Naik ${ }^{2 *}$, Naveed Ahmed ${ }^{3}$, Shahana Ayub ${ }^{4}$, Fatima Bilal Motiwala ${ }^{5}$ and Rizwan Ahmed}

${ }^{1}$ Department of Neurology, Jinnah Post Graduate Medical Centre, Karachi, Pakistan

${ }^{2}$ Department of Psychiatry, NYU School of Medicine, New York, NY, USA

${ }^{3}$ Department of Radiology, Jinnah Post Graduate Medical Centre, Karachi, Pakistan

${ }^{4}$ Agha Khan University Hospital, Karachi, Pakistan

${ }^{5}$ Jinnah Post Graduate Medical Centre, Karachi, Pakistan

\begin{abstract}
We report on 2 cases of Neurosarciodosis, it is a rare disease, primary care physicians and neurologists have very limited exposure to this pathology, published data till date is limited to few case series and case reports. There is also lack of enough demographical data of this illness in different regions around the globe, similarly there is absence of definitive diagnostic criterion for neurosarcodosis, and so it is often misdiagnosed and overlooked by clinicians due to identical clinical findings in other pathologies. This paper will review the presentation of two case reports and also highlight atypical presentations of this pathology that could potentially an aid to literature till date.
\end{abstract}

Keywords: Neurosarcoidosis; Noncaseating granulomas; Steroids; MRI

Abbreviations: ESR: Erythrocyte Sedimentation Rate; LFTs or LFs: Liver Function Tests; ACE: Angiotensin-Converting-Enzyme; WBCs: White Blood Cells

\section{Introduction}

Sarcoidosis is a multisystem inflammatory granulomatous disease with undetermined etiology, usually characterized by noncaseating granulomas in multiple organs, the respiratory and lymphatic systems are most commonly involved, but less frequently affects nervous system 5-15\% [1,2]. Within Nervous system, facial nerve dysfunction commonly occurs more than 50\% [3] followed by Parenchymal brain lesions about $50 \%$ of cases, and spinal involvement occurs in less than $10 \%$ of cases $[1,2,4] .1 \%$ of cases may present as an isolated neurological disease without features of systemic sarcoidosis [5]. A symptomatic neurosarcoidosis is present in about $10 \%$ of sarcoidosis patients [6] particularly, more than $50 \%$ of the patients who develop neurosarcoidosis become symptomatic within two years of diagnosis with systemic sarcoidosis [1,7]. The signs and symptoms disease are nonspecific and varied, commonly include cranial neuropathies, peripheral neuropathies, weakness, sensory disturbance, meningeal irritation, hydrocephalus, cognitive dysfunction, and personality changes, headache, visual disturbances, seizures, fatigue, nausea and vomiting $[1,2,8,9]$ and unexpected death [10]. Definitive diagnosis of neurosarcoidosis can be challenging [1,2], many other diseases presents with identical clinical findings should be excluded first, included brain and spinal cord tumors, inflammatory myelitis, tuberculosis meningitis and multiple sclerosis. An MRI is often chosen as a test choice, due to its overwhelming accurate ability to diagnose neurosarcoidosis. The MRI shows leptomeningeal enhancement, enhancement of cranial nerves, parenchymal lesions and hydrocephalus. In the absence or contraindication of a MRI, a CT scan of the head can be used as an alternative option. CSF abnormalities like lymphocytic pleocytosis and increased protein level are often seen in most of cases of neurosarcoidosis, though these findings are nonspecific for diagnosis [2]. The CSF angiotensin-converting enzyme level is also obtained but that is also nonspecific to make diagnosis neurosacrodoisis, CSF ACE level has low yield and may be false positive in course of malignancy and tuberculosis [11].

As seen in a commonly used diagnostic criterion by Zajicek et al.
[12] which states that 'probable neurosarcoidosis, requires clinical presentation suggestive of neurosarcoidosis, and the exclusion of other causes of neurologic dysfunction. Pathologic confirmation of systemic sarcoidosis and positive results for at least two of the following: gallium scan, chest high-resolution computed tomography (HRCT), BAL with a CD4:CD8 ratio of more than 3.5 and a CD4:CD8 ratio of more than 5 in the CSF $[12,13]$.

To best our knowledge within the published data, there is absence of a randomized double blind controlled trial for treatment of Neurosarcodosis, although immunosuppressive therapy is used effectively to prevent irreversible neurological deficits, corticosteroid therapy is widely used for symptomatic neurosarcodidos [2], but high doses steroids are only used for severe symptomatic cases, however for long-term treatment an alternative option is immuno suppressive therapy, that is a better choice in substitute of steroids, that include infliximab, azathioprine, methotrexate, hydroxychloroquine, cyclophosphamide.

\section{Methods}

Paper reporting patients those are diagnosed as a neurosarcoidosis, the diagnosis of neurosarcoidosis is based on diagnostic criteria proposed by Zajicek et al. [12].

\section{Clinical Presentation}

\section{Case 1}

A 25 years male presented to Emergency Department with complaint of headache and altered consciousness for 3 days; he has nausea, vomiting without visual or behavioral problem. He has history

*Corresponding author: Savita Naik, Department of Psychiatry, NYU School of Medicine, New York, NY 145 East $32^{\text {nd }}$ street, New York, NY, USA, Tel: 201-6876749; E-mail: savitanaik2000@gmail.com

Received August 22, 2013; Accepted September 17, 2013; Published September 20, 2013

Citation: Kumar S, Ahmed S, Naik S, Ahmed N, Ayub S, et al. (2013) Neurosarcoidosis: An Overlooked Diagnostic Dilemma. J Neurol Disord 1: 132 doi:10.4172/2329-6895.1000132

Copyright: ๑ 2013 Kumar S, et al. This is an open-access article distributed under the terms of the Creative Commons Attribution License, which permits unrestricted use, distribution, and reproduction in any medium, provided the original author and source are credited. 
Citation: Kumar S, Ahmed S, Naik S, Ahmed N, Ayub S, et al. (2013) Neurosarcoidosis: An Overlooked Diagnostic Dilemma. J Neurol Disord 1: 13ロ doi:10.4172/2329-6895.100013!

of on and off seizures for 3 years, seizures usually occur during middle of night, type of seizures was generalized tonic clonic associated with tongue bite and urine incontinence, due to these clinical findings patient was diagnosed with Tuberculosis Meningitis by a General Practitioner and started on anti-tuberculosis treatment for 18 months, despite of taking Anti-tuberculosis treatment, patient continued to develop seizures and consulted with another physician, who diagnosed him as an Epilepsy of unknown etiology. Patient was started on antiepileptic drugs but still he remained symptomatic and continued to develop seizures, and finally presented to this hospital with complaint of headache, seizures and altered consciousness.

On arrival of patient, MRI and CSF study of patient were done to rule out any suspected malignancy or chronic inflammatory disease, MRI showed ill-defined abnormal signal intensity areas involving the bilateral periventricular region, additionally post contrast sagittal images shows nodular meningeal enhancement more marked along the basal meninges and tentorium that is highly suggestive of granuloma, CSF study showed protein $164 \mathrm{mg} \%$, WBC 20 (95\% Lymphocytes), normal glucose and negative for India ink and cryptococcal antigen, after getting these findings we decided to order Chest X-ray followed by confirmatory HCRT, HRCT showed nodular thickening along with bronchovascular interstitium, multiple enlarge lymph nodes in right axilla \& mediastinum, these finding strongly suggestive of sarcoidosis (Figure 1).

\section{Case 2}

25-year-old male presented to Emergency Department with decreased vision, recurrent bilateral lower limbs weakness, and urinary incontinence, On Neurological examination, patient has power 3/5, exaggerated reflexes in lower limbs with Up-going planters, with normal upper limbs. Due to Eye Symptoms patient was urgently referred to ophthalmologist for eye examination that showed old chorioretinal scar and posterior synachie formation in anterior chamber of eye suggestive of posterior uveitis, but without keratic precipitates, due to neurological symptoms and other presenting complaints, CSF study was done that showed proteins of $121 \mathrm{mg} \%$, WBC 61 (90\% Lymphocytes), India ink and cryptococcal antigen was negative. In a similar manner other
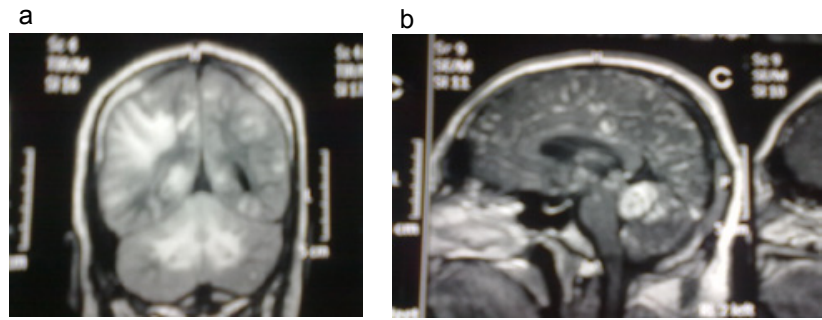

C

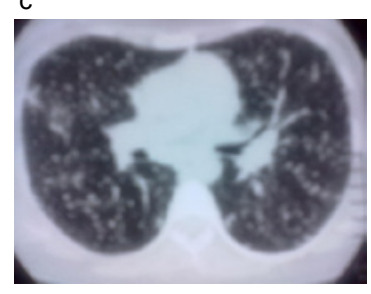

Figure 1: MRI Brain (a) Abnormal signal intensity areas seen involving the bilateral periventricular region, in Coronal Flair. MRI Brain (b) post contrast sagital images shows nodular meningeal enhancement more marked along the basal meninges and tentorium Suggestive of Granuloma. (c) HRCT chest showing nodular thickening along bronchovascular interstitium suggestive of Sarcoidosis Bronchovascular Interstitium.
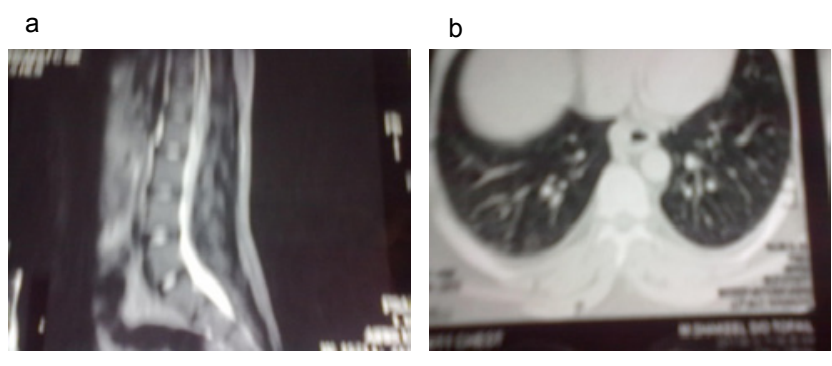

Figure 2: (a) MRI Sagital fat saturated contrast T1 weighted MRI showing enhancement of nerve roots suggestive of Granuloma. (b) HRCT chest showing mild nodular thickening along fissures with node in right para esophageal region.

baseline tests were also done, in which ESR was found raised while serum ACE levels, Complete Blood picture, Serum creatinine, and LFTs were normal. ANA was also done that appeared negative. Due to suggestive Clinical and CSF findings, a MRI with contrast of the brain was ordered, that showed T1 weighted Sagittal fat saturated contrast shows enhancement of nerve roots, and $\mathrm{T} 1$ weighted post contrast fat saturated coronal image shows nodular enhancement of nerve root on left side suggestive of granuloma. Clinical findings, CSF report and MRI results suggested provisional diagnosis of Neurosarcodsis, by following these results HRCT was done that showed bilateral hilar lymphadenopathy and interstitial fibrosis of both lungs (Figure 2).

\section{Discussion}

This paper describes our experience with 2 cases of Neurosarciodosis at JMPC Karachi. Since it a rare disease, neurologists have very limited exposure to deal with these cases, published data till date is limited to few case series and case reports, similarly these is absence enough demographical evidence of incidence and prevalence of neurosarcoidosis in the region. Regardless of neurological involvement, systemic sarcoidosis is often overlooked in primary care settings due to non-significant findings on chest $\mathrm{x}$-ray and laboratory tests. Therefore, a CT of the chest should be done on high clinical suspicion and moreover extensive workups like a MRI of the brain should be performed for patients who present with neurological symptoms as seen in our Case\#01. During the course of disease, cranial nerves involvement like facial or optic nerve, and the symptoms that arise due to their dysfunction could be initial presentation of patient as in case $\# 1$. The clinical diagnosis of neurosarcoidosis is fairly uncomplicated when a known case of systemic sarcoidosis presents with neurological symptoms, however it becomes challenging when it presents with lone neurological involvement as our case \#1 and 2. Case 1 and 2 presented with lone neurological symptoms without evident manifestation of systemic sarcoidosis, such presentation often misdiagnosed in primary care setting particularly in 3rd world countries due to limitations of performing extensive workup due to financial constrains. The differential diagnosis of neurosarcodisis covers broad list of diseases such as optic neuropathy, Bell's palsy, tuberculosis meningitis, CNS vasculitis, metastatic tumors, and CNS lymphomas, and the clinical presentation neurosarcoidosis is identical to these pathologies, subsequently It is vital to be aware of early clinical manifestations and act accordingly, which can be essential in allowing it to be differentiated from other diseases. The correct diagnosis and early treatment with steroid therapy could decrease the morbidity as well as it shows remarkable response in follow up MRI of brain. Both patients were treated with steroids during hospitalization and they responded very 
Citation: Kumar S, Ahmed S, Naik S, Ahmed N, Ayub S, et al. (2013) Neurosarcoidosis: An Overlooked Diagnostic Dilemma. J Neurol Disord 1: 13凸. doi:10.4172/2329-6895.100013!

well. Patients were scheduled and discharged on prednisone $0.5 \mathrm{mg} / \mathrm{kg}$ per day for four weeks follow-up.

\section{References}

1. Terushkin V, Stern BJ, Judson MA, Hagiwara M, Pramanik B, et al. (2010) Neurosarcoidosis: presentations and management. Neurologist 16: 2-15

2. Hoitsma E, Drent M, Sharma OP (2010) A pragmatic approach to diagnosing and treating neurosarcoidosis in the 21st century. Curr Opin Pulm Med 16: $472-479$

3. Pawate S, Moses H, Sriram S (2009) Presentations and outcomes of neurosarcoidosis: a study of 54 cases. QJM 102: 449-460.

4. Hamzeh N (2011) Sarcoidosis. Med Clin North Am 95: 1223-1234.

5. Vargas DL, Stern BJ (2010) Neurosarcoidosis: diagnosis and management Semin Respir Crit Care Med 31: 419-427.

6. Stern BJ, Krumholz A, Johns C, Scott P, Nissim J (1985) Sarcoidosis and its neurological manifestations. Arch Neurol 42: 909-917.
7. Agbogu BN, Stern BJ, Sewell C, Yang G (1995) Therapeutic considerations in patients with refractory neurosarcoidosis. Arch Neurol 52: 875-879.

8. Gascón-Bayarri J, Mañá J, Martínez-Yélamos S, Murillo $O$, Rene R, et al (2011) Neurosarcoidosis: report of 30 cases and a literature survey. Eur $J$ Intern Med 22: e125-e132.

9. Stern BJ (2004) Neurological complications of sarcoidosis. Curr Opin Neurol 17: $311-316$

10. Byard RW, Manton N, Tsokos M (2008) Sarcoidosis and mechanisms of unexpected death. J Forensic Sci 53: 460-464.

11. Dale JC, O'Brien JF (1999) Determination of angiotensin-converting enzyme levels in cerebrospinal fluid is not a useful test for the diagnosis of neurosarcoidosis. Mayo Clin Proc 74: 535

12. Zajicek JP, Scolding NJ, Foster O, Rovaris M, Evanson J, et al. (1999) Centra nervous system sarcoidosis--diagnosis and management. QJM 92: 103-117.

13. Marangoni S, Argentiero V, Tavolato B (2006) Neurosarcoidosis. Clinical description of 7 cases with a proposal for a new diagnostic strategy. J Neurol 253: 488-495. 